\title{
Erratum to: CT exposure in adult and paediatric patients: \\ a review of the mechanisms of damage, relative dose and consequent possible risks
}

\section{Stefano Colagrande $\cdot$ Daniela Origgi $\cdot$ \\ Giovanna Zatelli • Andrea Giovagnoni · Sergio Salerno}

Published online: 9 April 2014

(C) Italian Society of Medical Radiology 2014

\section{Erratum to: Radiol med}

\section{DOI 10.1007/s11547-014-0393-0}

In the online version of the article the following equation is incorrect:

$\mathrm{CTDI}_{\mathrm{w}}=2 / 3 \mathrm{CTDI}_{100, \mathrm{c}}+2 / 3 \mathrm{CTDI}_{100, \mathrm{p}}$

The correct equation should read:

$\mathrm{CTDI}_{\mathrm{w}}=1 / 3 \mathrm{CTDI}_{100, \mathrm{c}}+2 / 3 \mathrm{CTDI}_{100, \mathrm{p}}$

The online version of the original article can be found under doi:10.1007/s11547-014-0393-0.

\section{S. Colagrande $(\square)$}

Dipartimento di Scienze Biomediche Sperimentali e Cliniche, Struttura Complessa di Radiodiagnostica (SOD 2),

Università di Firenze, Florence, Italy

e-mail: stefano.colagrande@unifi.it

S. Colagrande

Azienda Ospedaliero-Universitaria di Careggi,

Largo Brambilla 3, 50134 Florence, Italy

D. Origgi

Dipartimento di Fisica Medica, Istituto Europeo di Oncologia, Via Ripamonti 435, 20141 Milan, Italy

G. Zatelli

Dipartimento di Fisica Sanitaria, Azienda USL 10 Firenze,

Via di San Salvi 12, 50135 Florence, Italy

\author{
A. Giovagnoni \\ Sezione di Scienze Radiologiche Ospedali Riuniti, Dipartimento \\ di Scienze Cliniche Specialistiche e Odontostomatologiche, \\ Università Politecnica Marche, Torrette, Ancona, Italy \\ S. Salerno \\ Dipartimento di Biotecnologie e Biotecnologie Mediche e \\ Forensi, Università di Palermo, AOUP Policlinico, Via del \\ Vespro 127, 90127 Palermo, Italy \\ e-mail: sergio.salerno@unipa.it
}

